\title{
Development and Validation of the Hyperlipidemia
}

\author{
Attitudes and Beliefs in Treatment (HABIT) Survey for Physicians \\ Kathleen A. Foley, PhD, Joseph Vasey, PhD, Charles M. Alexander, MD, Leona E. Markson, ScD
}

\begin{abstract}
INTRODUCTION: Many patients treated with lipid-lowering medications in clinical practice do not achieve targeted National Cholesterol Education Program (NCEP) goals for low-density lipoprotein cholesterol (LDL-C), despite the proven efficacy of these medications. Understanding physician attitudes and beliefs about treating patients to goal may be useful in improving patient care and ensuring that all patients receive the benefits of treatments shown to be optimal in clinical trials.
\end{abstract}

OBJECTIVE: To develop a theoretically based, and statistically reliable and valid survey instrument for measuring the attitudes and beliefs of physicians toward hyperlipidemia and its treatment, including treatment of patients to goal. To determine whether the attitudes measured were associated with physician intentions to treat patients to LDL-C goal.

METHODS: We assessed the reliability of the instrument through an examination of the internal consistency and factor structure of the constructs. Validity was assessed through zero-order correlations among the constructs and the relationship between the constructs and an intent to treat to goal case study.

RESULTS: Internal consistency scores for the 8 constructs ranged from 0.48 to 0.75 . Factor loadings indicated that the individual items belonged to their respective constructs, as hypothesized. The predictive validity of the instrument was demonstrated by significant relationships between 5 of the 8 attitudinal constructs and an intent to treat to goal case study.

CONCLUSIONS: The HABIT physician survey is the first validated instrument covering a broad set of attitudes about the treatment of hyperlipidemia that are both theoretically and empirically linked to physician intent to treat to NCEP LDL-C goal.

KEY WORDS: physician behavior; education; attitudes; cholesterol.

J GEN INTERN MED 2003; 18:984-990.

$\mathrm{H}$ igh cholesterol or hyperlipidemia is one of the major modifiable risk factors for coronary heart disease, which continues to be the leading cause of death and morbidity in the United States. ${ }^{1}$ Despite treatment guidelines and the wide-spread availability of pharmacological agents

Requests for the HABIT Physician Instrument should be addressed to Kathleen A. Foley.

Received from Outcomes Research \& Management, US Medical \& Scientific Affairs (KAF, CMA, LEM), Merck \& Co., Inc., West Point, Pa; and Center for Health Care and Policy Research (JV), Pennsylvania State University, University Park, Pa.

Address correspondence and requests for reprints to Dr. Foley: Merck \& Co., Inc., PO Box 4, WP39-170, West Point, PA 19486 (email:kathleen_foley@merck.com). proven to be efficacious in significantly lowering cholesterol levels and mortality in randomized clinical trials (RCTs), ${ }^{2-11}$ many patients are not treated to National Cholesterol Education Program (NCEP) goals for low-density lipoprotein cholesterol (LDL-C). ${ }^{12-16}$ One reason for lack of goal attainment may be that treatment choices made in clinical practice incorporate provider and patient attitudes, beliefs, and preferences that influence the effectiveness of pharmacological treatments. ${ }^{17,18}$ While the role of attitudes and beliefs in the treatment of hyperlipidemia has received limited attention in the literature, ${ }^{19,20}$ diabetes and hypertension researchers have begun to pay increasing levels of attention to how physician attitudes and beliefs influence patient outcomes. $^{21-23}$ Some evidence suggests, for example, that physician attitudes about treatments are more important determinants of patient outcomes than is physician knowledge. ${ }^{24,25}$ Findings from these studies regarding the important role of physician awareness of guidelines, feelings of inertia, and lack of self-efficacy in helping their patients meet their treatment goals are echoed in a recent review of the barriers to guideline adoption. ${ }^{26}$ A validated survey instrument assessing physician attitudes toward hyperlipidemia and its treatments should be an important component of a multipronged approach to improving the delivery of optimal patient care and lipid management.

Clearly, treating hyperlipidemic patients to their LDL-C goals is a multifactorial problem involving physician factors, patient factors, and health system factors. The HABIT physician survey addresses one piece of this puzzle by assessing physician attitudes and resource constraints that may impact on clinical decision making. The purpose of this study was to develop a statistically reliable and valid survey instrument for measuring the attitudes and beliefs of physicians toward hyperlipidemia and its treatment, including treatment of patients to goal. Our framework for developing the survey was based on the theory of reasoned action, which hypothesizes that individual attitudes toward particular behaviors, as well as the norms or social rules of the environment in which the individual lives, shape an individual's intention to behave in a particular way. ${ }^{27}$ This theory conceptually links a person's beliefs to their attitudes and behavior. An exploratory goal of this validation study was to determine whether the attitudes being measured were indeed associated with physician intentions to treat patients to LDL-C goal.

\section{METHODS}

\section{Survey Development}

The conceptual foundation of the survey was established on the basis of a literature review of physician attitudes, 
beliefs, and behaviors regarding the treatment of hyperlipidemia and other chronic conditions such as diabetes and hypertension. In reviewing the literature, we specifically looked for attitudinal factors that would be related to physician treatment decisions about patients currently being treated for hyperlipidemia. Our review identified 8 constructs or attitudes hypothesized to be related to treatment decisions. These 8 constructs are grouped into 3 overall themes, including attitudes about statins, attitudes about hyperlipidemia as a disease, and attitudes related to the context in which hyperlipidemia is treated.

Attitudes About Statins. Physician beliefs about the effectiveness of statins and other pharmacological treatments were identified as potential drivers of physician practices and treatment decisions. ${ }^{28,29}$ It is hypothesized that physicians who doubt the efficacy of statins or their effectiveness in particular patient groups will be less inclined to aggressively treat LDL-C. Two constructs related to dose titration of statins were also suggested by the literature to influence physician decisions. Physicians believing that patients view titration negatively may be less likely to titrate statin doses upward, due to a concern about communicating that the current treatment or the patient has somehow failed. In this situation, physicians may make decisions based on their perceptions of the patient's psychological responses to the treatment process. ${ }^{30}$ In addition, physicians concerned about the risks associated with higher statin doses may try other treatment alternatives such as encouraging diet and exercise, adding nonstatin agents, or avoiding higher statin doses altogether rather than titrating statin doses as recommended by NCEP guidelines. ${ }^{21,31,32}$

Attitudes About Hyperlipidemia as a Disease. Physician behaviors in treating hyperlipidemia may also be driven by their attitudes about hyperlipidemia overall and relative to other chronic conditions. Research on the treatment of hypertension, another chronic disease with national guidelines for treatment and goal attainment, suggests that some physicians may be satisfied with getting their patients close enough to goal rather than treating patients aggressively to goals as specified by the guidelines. ${ }^{33}$ Physicians treating hyperlipidemia may also believe that getting a patient to within 10 or 20 points of nationally recommended LDL-C goals is sufficient, and may therefore be less aggressive in their treatment decisions. At a more global level, physician attitudes about the urgency of getting to goal could impact on treatment decision making. ${ }^{22,29}$ Physicians may not see a need to get patients to goal quickly, given that hyperlipidemia is a chronic condition requiring life-long treatment as well as for a variety of other reasons, including the asymptomatic nature of hyperlipidemia and patient focus on other conditions.

Attitudes Related to the Context in Which Hyperlipidemia is Treated. Our review of the literature suggested 3 elements of the health care context that might impact on physician decision making about the pharmacological treatment of hyperlipidemia. First, decisions about pharmacological treatments may be influenced by a physician's sense of the utility of diet and exercise in lowering LDL-C. ${ }^{34-36}$ Physicians who have a positive attitude toward diet and exercise may be less aggressive in their use of pharmacological agents and vice versa. Second, physicians must make decisions within health systems that are subject to constraints on the time and resources available to counsel patients. ${ }^{29,37-39}$ Physicians' sense of the time and resources available to them may make them less likely to aggressively treat patients with pharmacological agents if they do not feel they have the time to counsel with regard to the benefits and risks of such treatment. Finally, a physician's sense of their own self-efficacy in treating patients to goal may be an important contributor to the treatment choices they make. ${ }^{22,29,34,38,39}$

Item Development. Individual questions and attitudinal statements for each of the above constructs were written in an iterative process by the authors who represent various disciplines including medicine, medical sociology, health services research, and psychometrics. In most cases, items were constructed to reflect the attitudes and beliefs of the physician relative to hyperlipidemia treatment. Response categories used a 5-point Likert scale to assess the degree to which physicians agreed or disagreed with each of the attitudinal statements.

Each attitudinal statement was evaluated with regard to whether it was an unambiguous and clearly stated example of the construct it represented. This activity resulted in the rewording, and sometimes the elimination, of poorly or awkwardly worded items, or items that were judged to be poor representatives of their constructs. The items and constructs, as a whole, were also reviewed in terms of response scaling, the direction of wording, transitional statements between survey sections, instructions to interviewers, item sequencing, and readability. The final survey was pilot tested on several physicians as a final check on its readability and to identify any problematic items or transitions. The Flesch-Kincaid grade level available in Microsoft Word (Microsoft Corporation, Redmond, WA) rated the final survey at an 8th grade reading level.

Attitudinal Scale Construction. Each of the attitudinal scales was calculated as the average of the individual item scores. All scales were scored so that they represent stronger agreement with the attitude. Thus, some items within each scale may have been reverse coded.

\section{Statistical Analyses}

Construct Validity. The term construct validity is used here to refer to the internal consistency (often referred to as reliability) and the factorial structure of the survey instruments. Taken together, these concepts describe how survey items relate to underlying factors and to one another. 
Internal consistency (homogeneity) is concerned with the internal relationships or consistency among the items that purportedly measure the same construct. This property of the HABIT scales was assessed through the use of Cronbach's a.

The internal consistency analyses were supplemented with a detailed examination of the factor structure of the survey. The overall adequacy of the survey's operationalization was assessed with a confirmatory factor analysis based on the intended or theoretical design. We examined the factor loadings from the confirmatory factor analysis to represent the degree to which the construct determines the response to an item and whether the individual attitudinal statements belonged to the constructs as we defined them.

Concurrent and discriminant validity were evaluated via the zero-order correlations among the scales. We assessed predictive validity by the instrument's ability to distinguish between physicians who had a high intent to treat patients to goal versus those who had a lower intent to treat to goal. Intent to treat patients to NCEP goal was measured via a case study in which physicians were asked to recommend treatment options for a patient exhibiting various lipid levels throughout his course of statin treatment. This case study allows for treatment decisions that are consistent with NCEP ATP III recommendations and studies of clinical practice. ${ }^{40}$ Specifically, physicians were asked to consider their treatment of a 60-year-old male with established CHD who they were seeing for the first time. The physician is assumed to order a routine lipid profile, which determines that the patient has an initial LDL of $190 \mathrm{mg} / \mathrm{dL}$. A statin medication is prescribed. The physician is asked what he/ she would do 1) after 8 weeks on the statin (LDL-C = 140); 2 ) in another 8 weeks (LDL-C = 125); and 3) in yet another 8 weeks (LDL-C $=110$ ). At each of the 3 points in time the physician can choose from 5 options: encourage diet and exercise, and re-evaluate in 12 to 16 weeks; encourage diet and exercise, and re-evaluate in 6 to 8 weeks; switch to another statin, add niacin or a fibrate; or increase the statin dose. The response choices were ordered from the least aggressive to the most aggressive treatment option for attaining LDL-C goal. Scores across the three 8-week scenarios were summed, with higher scores indicating a stronger intent to treat to goal. Scores ranged from 3 to 15 , with a median score of 14 . Scores of 14 and 15 were categorized as a high intent to treat to goal, while scores between 3 and 13 were categorized as low intent to treat to goal.

\section{Study Sample}

A sample of 400 physicians who had participated in previous cardiovascular or diabetes-related observational studies was invited to complete the questionnaire on paper or by telephone interview. Two hundred and three physicians completed the questionnaire, for a response rate of $51 \%$, which is consistent with other validation studies. ${ }^{25}$ Two questionnaires were excluded from the data analysis because they were returned after the deadline. Physician respondents included 78 cardiologists, 10 endocrinologists, 53 providers in internal medicine, 53 general or family practitioners, 5 providers in multiple specialty practices, and 2 who did not indicate their specialty background. On average, the physicians had been in practice for a mean of 19 years (standard deviation $[\mathrm{SD}]=8.2$ years), with a minimum of 2 years and a maximum of 50 years.

\section{RESULTS}

\section{Construct Validity}

The results of 2 statistical tests used to determine whether the individual question items were related to the attitude constructs as hypothesized are shown in Tables 1 and 2 . Reliability coefficients for the 8 scales range from 0.48 to 0.75 with between 2 and 6 items per scale (Table 1 ). The factor loadings for each question item were highest with the attitudes shown in Table 2, indicating that the individual items belonged to their respective constructs, as hypothesized.

\section{Concurrent and Discriminant Validity}

Table 3 shows the zero-order correlations between scale scores. The relationships between scales are in the expected directions and the majority of the correlations between constructs are low, with the exception of several high correlations that would be theoretically expected. Physicians who have a strong belief in the effectiveness of statins, for example, are less likely to believe that there are risks associated with higher doses $(r=-.54)$. Similarly, physicians who believe that patients view titration

Table 1. Internal Consistency (Reliability) Analysis of the Physician Survey

\begin{tabular}{|c|c|c|c|}
\hline Attitudinal Construct & Items, $N$ & Coefficient alpha & Mean (SD) \\
\hline 1. Statin effectiveness & 3 & 0.58 & $3.5(0.89)$ \\
\hline 2. Patients view titration negatively & 2 & 0.74 & $2.3(1.0)$ \\
\hline 4. Close enough to goal & 3 & 0.54 & $2.7(0.86)$ \\
\hline 5. Urgency of getting to goal & 2 & 0.48 & $3.6(0.99)$ \\
\hline 6. Utility of diet and exercise & 4 & 0.59 & $2.3(0.74)$ \\
\hline
\end{tabular}


Table 2. Individual Attitudinal Items and Factor Loadings

Effectiveness of Statins

1. Statin monotherapy is sufficient for getting most patients to goal.

2. Current options for treating hyperlipidemia do not get the majority of patients to their LDL goal.

3. Given patient variations in response to statins, many patients do not achieve

the expected efficacy in LDL lowering with statin monotherapy.

Patients View Titration Negatively

4. Increasing a patient's statin dose makes patients think the first dose was not correct.

5 . When a patient's dose is increased, the patient views his treatment as a failure.

Risks Associated with Higher Doses

6. Some patients are not able to tolerate high-dose statins.

7. The use of higher dose statins requires caution given increased safety risks.

8. The additional monitoring necessary with higher dose statins is not convenient for many patients.

0.651

9. Doubling the dose of a statin provides minimal incremental efficacy in LDL lowering.

0.482

10. Many patients will stop taking their statin medication if the dose is increased.

0.640

11. Patients prefer lower doses of statins.

Close Enough to Goal

12. Lowering a patient's LDL to within 10 points of goal is sufficient for

reducing the risk of future cardiac events.

13. LDL goals, as defined by standard guidelines, should be viewed as

suggestions rather than definitive goals for all patients.

14. An LDL of 120 is close enough to goal to prevent future heart

0.656

problems in CHD patients.

Urgency of Getting to Goal

15. Lowering LDL is often of secondary importance compared to other health

conditions that many patients have.

16. Achieving aggressive treatment goals in a short time period is not a

major focus of treatment for chronic conditions like hyperlipidemia.

Utility of Diet and Exercise

17. Most patients lack the motivation to make lifestyle changes.

18. Patients may attempt to improve their diet and exercise, but few succeed.

19. Starting treatment with diet and exercise delays the initiation of more effective treatment.

0.229

20. Initiating with diet and exercise increases the number of office

0.253

visits and time required to treat to goal.

Time and Resources to Counsel Patients

21. In general, how often did you have the time to fully discuss your patients'

diet and exercise programs for lowering cholesterol?

22. In general, how often did you have the time to fully address other

preventive health care issues with your patients?

23. In general, how often did you have the time to fully discuss the

severity of high cholesterol with your patients?

24. In general, how often did you have the resources you needed,

such as nurse or physician assistant time, and educational materials or

programs, to help your patients improve their diet, exercise, and other

preventive health measures?

Physician Self-efficacy in Treating Patients to Goal

25. Patients require more time than I can give to make lifestyle changes.

26. Most of what is needed to get patients to their LDL goal is outside of my control.

0.562

27. I have the time and resources necessary for bringing the majority

0.762

of my patients to their LDL goal.

28. Managed care restrictions negatively impact on my ability to get patients to goal.

0.338

29. I'm satisfied with my ability to get my patients to their LDL goal.

negatively are also likely to agree that there are risks associated with higher statin doses $(r=.68)$. These correlations are to be expected given that these 3 attitudes all relate to statins. Despite these intercorrelations, however, the confirmatory factor analysis identified these 3 attitudes as distinct constructs. Similar patterns were found among the 2 attitudes related to hyperlipidemia as a disease and 2 of the attitudes related to the context of hyperlipidemia treatment. As above, these high correlations are expected, given that these attitudes are theoretically related, yet the results of the confirmatory factor analysis show that each measures a distinct construct. 
Table 3. Zero-order Correlations Among the Attitudinal Scales

\begin{tabular}{|c|c|c|c|c|c|c|c|}
\hline & Scale 1 & Scale 2 & Scale 3 & Scale 4 & Scale 5 & Scale 6 & Scale 7 \\
\hline 1. Effectiveness of statins & 1.0 & & & & & & \\
\hline 2. Patients view titration negatively & -0.371 & 1.0 & & & & & \\
\hline 4. Close enough to goal & 0.175 & -0.022 & 0.219 & 1.0 & & & \\
\hline 5. Urgency of getting to goal & 0.250 & -0.256 & -0.395 & -0.638 & 1.0 & & \\
\hline 6. Utility of diet and exercise & 0.252 & -0.322 & -0.300 & -0.073 & 0.113 & 1.0 & \\
\hline
\end{tabular}

\section{Predictive Validity}

The results of the case study provide evidence for the predictive validity of the instrument (Table 4). High intent to treat to NCEP goal was significantly associated with less agreement that titration is not worth the risks; a lack of agreement with the attitude that close enough to goal is good enough; a stronger belief in the urgency of getting to goal; a greater availability of time and resources to counsel patients; and a stronger sense of self-efficacy in treating hyperlipidemia.

\section{DISCUSSION}

Recent studies point out that physician knowledge of guidelines is high and therefore explains little of the variation in physician practice patterns. Attitudes, however, are believed to play a much greater role. ${ }^{22,23,41}$ This view is consistent with the theory of reasoned action, which states that an individual's attitudes are related to intentions to behave in particular ways. ${ }^{27}$ While physician attitudes and beliefs are just one component of the process of care that influences treatment decisions, they are an important component for several reasons. First, while some may argue that health system processes and constraints play a greater role than attitudes in driving patient outcomes, changing the health system is largely outside of individual control. Attitudes, on the other hand, can potentially be modified to encourage specific behaviors within the treatment context or improve physician-patient communication about hyperlipidemia. Furthermore, where attitudes are difficult to change, alternative treatment options may be identified that are consistent with the existing attitudes.
Previous efforts to measure physician attitudes about hyperlipidemia have been limited to assessments of the circumstances under which a physician would initiate statin therapy. ${ }^{19,20}$ The HABIT instrument, on the other hand, captures a much broader spectrum of attitudes related to the treatment of hyperlipidemia, including attitudes about the health care context in which treatment decisions are made. The HABIT physician survey can help improve patient care by enabling health plans and physicians to better understand attitudes that impact on the delivery of optimal care. Results from this survey could be used in medical education programs aimed at influencing physician behaviors or treatment practices. The survey will also be useful in future hyperlipidemia research by providing baseline information about physicians that can be used in statistical models (i.e., propensity scoring) to adjust for differential treatment choices.

The results of this validation study provide preliminary support for the reliability and validity of this first version of the HABIT attitudinal scales for physicians. The range in Cronbach's a from 0.48 to 0.75 across the 8 scales is similar to the range of 0.40 to 0.78 reported for the first version of the diabetes attitudinal scale, which also consisted of 8 subscales. ${ }^{25}$ Improvements in the reliability scores of each of the 8 scales are anticipated with future versions of the HABIT instrument derived from the results of this validation study. Evidence of the construct validity of the instrument was demonstrated by the large factor loadings between each item and its construct. The consistency of the findings from both the internal consistency and factor structure analyses provides additional support for the validity of the attitudinal constructs.

\section{Table 4. Predictive Validity-Differences in Mean Scores (Standard Deviation) of Physician Attitudes by Degree of} Intent to Treat to Goal

\begin{tabular}{|c|c|c|c|}
\hline & $\begin{array}{l}\text { High Intent Treat to Goal, } \\
\text { mean (SD) }\end{array}$ & $\begin{array}{l}\text { Lower Intent to Treat to Goal, } \\
\text { mean (SD) }\end{array}$ & $P$ Value \\
\hline 1. Effectiveness of statins & $3.52(0.85)$ & $3.34(0.89)$ & .1423 \\
\hline 2. Patients view titration negatively & $2.33(1.1)$ & $2.36(0.96)$ & .8265 \\
\hline 3. Risks associated with higher doses & $2.94(0.59)$ & $3.16(0.57)$ & .0105 \\
\hline 4. Close enough to goal & $2.51(0.88)$ & $2.78(0.80)$ & .0299 \\
\hline 5. Urgency of getting to goal & $3.85(0.87)$ & $3.37(0.99)$ & .0004 \\
\hline 6. Utility of diet and exercise & $2.23(0.80)$ & $2.34(0.70)$ & .3343 \\
\hline 7. Time and resources to counsel patients & $3.28(0.58)$ & $3.12(0.52)$ & .0468 \\
\hline 8. Physician efficacy in treating patients to goal & $3.63(0.68)$ & $3.23(0.79)$ & .0002 \\
\hline
\end{tabular}


Although our ability to fully test the validity of the instrument is limited by the lack of other attitudinal instruments and measures of physician behaviors, we were able to evaluate some measures of concurrent, discriminant, and predictive validity. While each of the scales is shown to represent a unique attitudinal construct, the constructs are related to one another in ways that would be theoretically expected. In addition, the statistically significant associations between 5 of the 8 attitudes (including risks associated with higher doses, close enough to goal, urgency of getting to goal, time and resources to counsel patients, and physician efficacy in treating patients to goal) and the intent to treat to goal case study provide strong evidence of the predictive validity of the HABIT physician survey. While additional tests of the validity of this instrument are clearly required, the evidence presented here provides strong support for the preliminary reliability and validity of the instrument.

This validation study was conducted with a sample of physicians who had participated in previous observational research related to hyperlipidemia and diabetes. The physician population is not necessarily generalizable to the broader population of physicians who treat hyperlipidemia in clinical practice. While the distribution of the attitude scales may vary in different physician populations, there is no theoretical reason to believe that the psychometric properties of the instrument would differ among populations. However, additional studies assessing the validity of the HABIT instrument as it relates to physician behaviors and treatment strategies in different physician populations would be valuable.

In general, the factors that contribute to the discrepancy between treatment efficacy obtained in clinical trials and treatment effectiveness found in clinical practice have not been very well documented in the existing literature. In the case of statin treatment for hyperlipidemia, however, studies on the lack of goal attainment suggest that physicians in clinical practice may not use treat to goal strategies, such as the titration-based strategy commonly used in RCTs, often enough to bring the majority of their patients to LDL-C goal. The results of this study suggest that physician attitudes about hyperlipidemia and its treatment are related to their intentions to treat patients to goal. Thus, physician attitudes, which randomization to treatment and strict treatment protocols attempt to remove in clinical trials, ${ }^{42}$ may partially explain why so many patients are not treated to goal in practice.

Physician attitudes about disease and treatments are just one part of a multifactorial problem that also includes patient and health system factors. In an effort to capture some of the health system factors that directly impact on physician behavior, the HABIT survey includes questions on whether physicians feel they have the time and resources necessary to address their patients, health needs. The results of this study suggest that inadequate time and resources are, in fact, associated with physician intentions to treat patients to goal. Furthermore, it is possible that physicians communicate their attitudes about goal attainment and the effectiveness of the drugs used to lower cholesterol to their patients. Thus, physician attitudes may also impact directly on patient adherence as well as be influenced by physician perceptions of patient adherence.

The HABIT physician survey is the first validated instrument covering a broad set of attitudes about the treatment of hyperlipidemia that are both theoretically and empirically linked to physician intent to treat to NCEP LDL$\mathrm{C}$ goal. This instrument provides a first step in assessing some of the attitudinal and systemic factors that act as barriers to getting patients to their treatment goals.

Does emerging research make our efforts to better understand goal attainment irrelevant? The Heart Protection Study (HPS) investigators have stated that they believe that high-risk patients (e.g., CHD and/or diabetes) should be treated with lipid medications regardless of baseline LDL cholesterol levels. ${ }^{11}$ However, it is not clear if and when NCEP guidelines will be changed as a result of HPS. ${ }^{15}$ Given the large numbers of high-risk patients not receiving optimal care under the current guidelines, even if NCEP guidelines are changed, the need for a better understanding of the attitudes and beliefs of physicians and patients that are likely to lead to optimal care will remain.

This research was funded by Merck/Schering-Plough Pharmaceuticals.

We acknowledge the reviewers for their helpful comments and suggestions for improving the manuscript.

\section{REFERENCES}

1. American Heart Association. 2001 Heart and Stroke Statistical Update. Dallas, TX: American Heart Association; 2000.

2. The Scandinavian Simvastatin Survival Study Group. Randomized trial of cholesterol lowering in 4444 patients with coronary heart disease. The Scandinavian Simvastatin Survival Study (4S). Lancet. 1994;344: 1383-9.

3. The Scandinavian Simvastatin Survival Study Group. Baseline serum cholesterol and treatment effect in the Scandinavian Simvastatin Survival Study. Lancet. 1995;345:1274-5.

4. Sacks FM, Pfeffer MA, Moye LA, et al. The effect of pravastatin on coronary events after myocardial infarction in patients with average cholesterol levels. Cholesterol and recurrent events trial investigators. N Engl J Med. 1996;335:1001-9.

5. The Long-Term Intervention with Pravastatin in Ischaemic Disease (LIPID) Study Group. Prevention of cardiovascular events and death with pravastatin in patients with coronary heart disease and a broad range of initial cholesterol levels. N Engl J Med. 1998;339:1349-57.

6. Goldberg RB, Mellies MJ, Sacks FM, et al. Cardiovascular events and their reduction with pravastatin in diabetic and glucoseintolerant myocardial infarction survivors with average cholesterol levels: subgroup analyses in the cholesterol and recurrent events (CARE) trial. Circulation. 1998;98:2513-9.

7. Lipid Research Clinics Program. The Lipid Research Clinics Coronary Primary Prevention Trial results. I. Reduction in incidence of coronary heart disease. JAMA. 1984;251:351-64.

8. Frick M, Elo O, Haapa K, et al. Helsinki Heart Study: primary prevention trial with gemfibrozil in middle-aged men with dyslipidemia. N Engl J Med. 1987;317:1237-45.

9. Shepherd J, Cobbe S, Ford I, et al. for the West of Scotland Coronary Prevention Study Group. Prevention of coronary heart disease with 
pravastatin in men with hypercholesterolemia. N Engl $\mathrm{J}$ Med. 1995;333:1301-7.

10. Downs J, Clearfield M, Weis S, et al. Primary prevention of acute coronary events with lovastatin in men and women with average cholesterol levels: results of AFCAPS/TexCAPS. JAMA. 1998;279:1615-22.

11. Heart Protection Study Collaborative Group. MRC/BHF Heart Protection Study of cholesterol lowering with simvastatin in 20,536 high-risk individuals: a randomized placebo-controlled trial. Lancet. 2002;360:7-22.

12. Olson K, Bungard T, Tsuyuki R. Cholesterol risk management: a systematic examination of the gap from evidence to practice. Pharmacotherapy. 2001;21:807-17.

13. Sueta C, Chowdhury M, Boccuzzi S, et al. Analysis of the degree of undertreatment of hyperlipidemia and congestive heart failure secondary to coronary artery disease. Am J Cardiol. 1999;83:1303-7.

14. Hyman D, Maibach E, Flora J, Fortmann S. Cholesterol treatment practices of primary care physicians. Public Health Rep. 1992;107:441-8.

15. Expert Panel on Detection, Evaluation, and Treatment of High Blood Cholesterol in Adults. Executive Summary of the Third Report of the National Cholesterol Education Program (NCEP) Expert Panel on Detection, Evaluation, and Treatment of High Blood Cholesterol in Adults (Adult Treatment Panel III). JAMA. 2001;285:2486-97.

16. Abookire S, Karson A, Fiskio J, Bates D. Use and monitoring of "statin" lipid-lowering drugs compared with guidelines. Arch Intern Med. 2001;161:53-8.

17. Black N. Why we need observational studies to evaluate the effectiveness of health care. BMJ. 1996;312:1215-8.

18. Ray J. Evidence in upheaval: incorporating observational data into clinical practice. Arch Intern Med. 2002;162:249-54.

19. Yarzebski J, Bujor CF, Goldberg RJ, Spencer F, Lessard D, Gore JM. A community-wide survey of physician practices and attitudes towards cholesterol management in patients with recent acute myocardial infarction. Arch Intern Med. 2002;162:797-804.

20. Superko H, Desmond D, de Santos V, Vranizan K, Farquhar J. Blood cholesterol treatment attitudes of community physicians: a major problem. Am Heart J. 1988;116:849-55.

21. Huse DM, Roht LH, Alpert JS, Hartz SC. Physician knowledge, attitudes and practice of pharmacologic treatment of hypertension. Ann Pharmacother. 2001;35:1173-9.

22. Larme A, Pugh J. Attitudes of primary care physicians toward diabetes. Diabetes Care. 1998;21:1391-6.

23. Anderson RM, Donnelly MB, Davis WK. Controversial beliefs about diabetes and its care. Diabetes Care. 1992;15:859-63.

24. Fix K, Oberman A. Barriers to following national cholesterol education program guidelines: an appraisal of poor physician compliance. Arch Intern Med. 1992;152:2385-7.

25. Anderson R, Donnelly M, Gressard C, Dedrick R. Development of diabetes attitude scale for health-care professionals. Diabetes Care. 1989;12:120-7.
26. Cabana M, Rand C, Powe N, et al. Why don't physicians follow clinical guidelines? JAMA. 1999;282:1458-65.

27. Ajzen I, Fishbein M. Understanding Attitudes and Predicting Social Behavior. Englewood Cliffs, NJ: Prentice Hall; 1980.

28. McBride P, Schrott H, Plane M, Underbakke G, Brown R. Primary care practice adherence to national cholesterol education program guidelines for patients with coronary heart disease. Arch Intern Med. 1998; 158:1238-44.

29. Green L, Eriksend M, Schor E. Preventive practices by physicians: behavioral determinants and potential interventions. Am J Prev Med. 1988;4(4 suppl):101-7.

30. Brett AS. Psychological effects of the diagnosis and treatment of hypercholesterolemia: lessons from case studies. Am J Med. 1991;91:642-7.

31. Andrade S, Walker A, Gottleib L, et al. Discontinuation of antihyperlipidemia drugs-do rates reported in clinical trials reflect rates in primary care settings? N Engl J Med. 1995;332:1125-31.

32. Aronow W. Underutilization of lipid-lowering drugs in older persons with prior myocardial infarction and a serum low-density lipoprotein cholesterol $>125 \mathrm{mg} / \mathrm{dL}$. Am J Cardiol. 1998;82:668-9.

33. Oliveria SA, Lapuerta P, McCarthy BD, L'Italien GJ, Berlowitz DR, Asch SM. Physician related barriers to the effective management of uncontrolled hypertension. Arch Intern Med. 2002;162:41320.

34. Valente CM, Sobal J, Muncie HL, Levine DM, Antlitz AM. Health promotion: physician's beliefs, attitudes and practices. Am J Prev Med. 1986;2:82-8.

35. Kottke T, Foels J, Hill C, Choi T, Fendersonet D. Nutrition counseling in private practice: attitudes and activities of family physicians. Prev Med. 1984;13:219-25.

36. Ammerman AS, DeVellis RF, Carey TS, et al. Physician-based diet counseling for cholesterol reduction: current practices, determinants and strategies for improvement. Prev Med. 1993;22:96-109.

37. Pearson T, Peters T. The treatment gap in coronary artery disease and heart failure: community standards and the post-discharge patient. Am J Cardiol. 1997;80(8B):45H-52H.

38. Shea S, Gemson D, Mossel P. Management of high blood cholesterol by primary care physicians: diffusion of the National Cholesterol Education Program Adult Treatment Panel guidelines. J Gen Intern Med. 1990;5:327-34.

39. Roberts W. Getting cardiologists interested in lipids. Am J Cardiol. 1993;72:744-5.

40. Foley K, Simpson R, Crouse J, Weiss T, Markson L, Alexander C. Effectiveness of statin titration on low density lipoprotein cholesterol goal attainment in patients at high risk of atherogenic events. Am J Cardiol. 2003;92:79-81.

41. Pearson T, Laurora I, Chu H, Kafonek S. The lipid treatment assessment project. Arch Intern Med. 2000;160:459-67.

42. Simon G, Wagner E, Vonkoff M. Cost-effectiveness comparisons using "real world" randomized trials: the case of new antidepressant drugs. J Clin Epidemiol. 1995;48:363-73. 